\title{
Damage detection in a steel frame using compressed EMI signatures based on compression sensing theory
}

\author{
Ji ZHENG ${ }^{1, a}$, Wei YAN ${ }^{1, b}$ \\ ${ }^{1}$ Faculty of Architectural, Civil Engineering and Environment, Ningbo University, Ningbo, Zhejiang, \\ 315211, China \\ a 413952152@qq.com, b yanwei4467@126.com
}

Keywords: Compression sensing; Electro-mechanical impedance (EMI); Steel Frame; Principal component analysis (PCA); Artificial neural network (ANN); Damage detection.

\begin{abstract}
An experiment was implemented using the electro-mechanical impedance (EMI) health monitoring technology for a framed structure and the conductance data were accordingly obtained for various damage severities. The compressed data by compression sensing theory were used to character the structural damages instead of the raw ones, and were further studied based on the principal component analysis (PCA). The obtained principal components were then employed to be the input parameters of the BP artificial neural network (ANN). Results showed that the transmission bandwidth and storage space of the EMI data were only $40 \%$ of the original ones using the present method. The neural network could identify the appearance of damages and could further classify the damage severities quantitatively using the principal components of the compressed conductance.
\end{abstract}

\section{Introduction}

Electro-mechanical impedance method has been demonstrated to be a potentially powerful structural damage detection technique. The identification of cracks, damages in metallic structures, loosening connections of pipelines, and delaminations in composites structures has been investigated by many researchers [1,2]. For an online real-time EMI structural health monitoring system, the measured data acquisition and processing are very important. The EMI signals should be transmitted timely to the system processor, which can then make a proper judgment for damage diagnose. However, in the practical engineering applications, the obtained EMI data are very large. In order to achieve efficient transmission and timely diagnosis function, the raw data should be compressed to reduce the computer memory.

On the other hand, the compression sensing theory [3,4] has attracted much attention in the field of signal processing. In this paper, the compression sensing technique will first be used for pre-treatment of measured EMI signatures to reduce the dimension of data. The basic idea of this method is the global observation to the sparse signal far below the Nyquist sampling rate rather than local sampling [5]. A principal component analysis (PCA)-based data reduction technique is further applied to the compressed EMI data. The compressed EMI data, represented by their projection onto the most significant principal components, are then used as ANN input variables instead of the pristine EMI data.

\section{Experiment and discussion}

A steel frame with bolt joints, as shown in Figures 1, is investigated. The piezoelectric patches are bonded on the critical section of certain joint of the frame and are connected with impedance analyzer 4294A by two wires. Damages are simulated by completely loosening bolts. In this experimental case, five conditions are taken into account: (1) Pristine; (2) loosening the No.1 bolt; (3) loosening the No.1 and No.2 bolts; (4) loosening the No.1, No.2 and No.3 bolts; (5) loosening the No.1, No.2, No.3 and No.4 bolts.

Figure 2 displays the real $(G)$ and imaginary part (B) of admittance for the experimental case. The piezoelectric admittance changes obviously with the increasing number of loosening bolts. This is because the structure damping and the dissipation of energy increase and conversely the structural 
stiffness of steel frame decreases. Therefore, EMI technology can identify structural damage well. However, Figure 2 just reflects the existence of damage qualitatively and can not assess damage severities quantitatively. Besides, in the practical applications, signal sampling points are numerous, the direct acquisition of signal transmission and storage to test system is very difficult. Therefore, this paper will put forward not only compress memory but also quantitative damage assessment method, which is based on compression sensing of EMI signatures and ANN structural damage identification method.
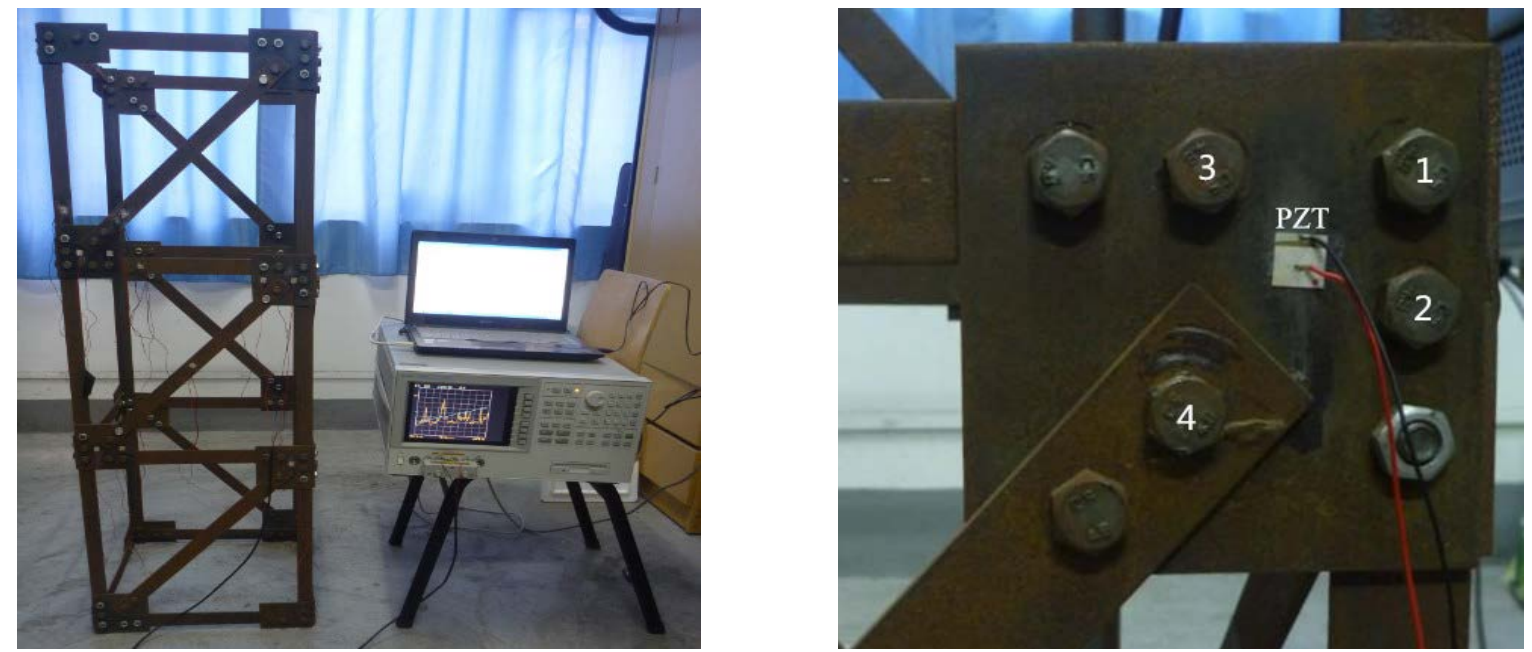

Figure 1. Experimental set up
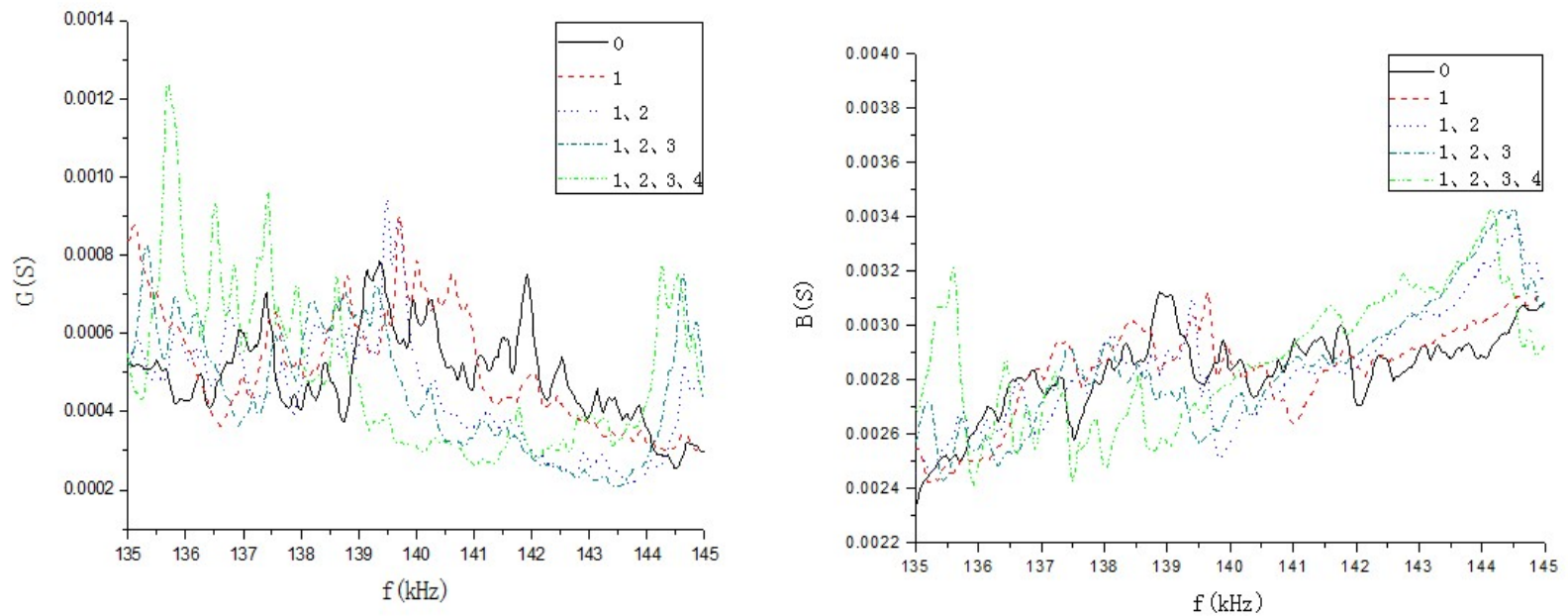

Figure 2. Admittance signatures for different damage severity

\section{The compression sensing of conductance signal}

Set $x$ is one dimensional signal, its length is $N$ and the sparse degree is $K . \Phi$ is the two-dimensional matrix $M \times N(M<N) \cdot y=\Phi x$ is one dimensional measurements value for the length of $M$. Compression sensing problem is to solve the underdetermined equations, and get the original signal $x$ based on known measured values $y$ and the observation matrix $\Phi$.

It is necessary to solve the optimization problem as follows:

$$
x=\arg \min \|x\|_{0} \quad \text { s.t } \Phi x=y
$$

It has showed that the number of observations is four times or above than that of the sparse degree, it can be realized that the signals are reconstructed without any distortion $[3,4]$. In the present experiment, five operating conditions are considered and meanwhile 3 sets data including conductance, susceptance and the modulus of admittance are taken to be the input parameters. Thus, 15 sets data should be taken into account. Therefore, once the conductance signal sparse degree $K$ is known, only $4 \times K$ time Gaussian random observations are necessary: 
$y=\Phi_{4 K \times 500} f_{500 \times 15}$

Because $y$ contains almost all of original data information, EMI health monitoring system only need to transmit and store the compressed signal $y$. We can use discrete cosine transform on sparse decomposition and determined the sparse degree, the steps as follows:

1. The discrete EMI conductance signal $f$ is obtained by simulation;

2. The discrete cosine transform (DCT) to $f$;

3. The absolute value of the transform domain component from big to small order;

4. $s=1$;

5. Calculate the total energy E before s components;

6 . When $\mathrm{E}$ is over $99.9 \%$ of all components of energy, stop the calculation, the sparse degree is s. Otherwise $s=s+1$ and go back to step 5 .

Through Matlab programming calculation of 15 different damage severities of conductance signal sparse degree, its value is 49 , so the sparse degree is taken to be $K=50$.

Currently, the widely used measurement matrix is a Gaussian random matrix. According to the 4 times sparse degree theory, only $200(4 \times 50)$ times Gaussian random observation can achieve the effect of compression:

$$
y_{200 \times 15}=\Phi_{200 \times 500} f_{500 \times 15}
$$

in which $\Phi_{200 \times 500}$ is the Gaussian random observation matrix.

Thus, when the number of observations is four times of the sparse degree, EMI damage detection only need 200 observations for transport and storage and can keep the damage information of the original signal, whose transmission bandwidth and storage space are only $40 \%$ of the original ones.

\section{Damage detection}

Principal component analysis (PCA) is a linear data compression technique which is widely used in the fields of image processing, flow visualization, pattern recognition and time-series prediction. It can be viewed as a statistical technique for achieving a dimensionality reduction. The advantage of PCA is to reduce the dimension of the input parameters of neural network, to remove redundant information and to optimize network structure. First, to normalized processing for the compressed conductance data. Then, PCA was carried out on the normalization of EMI signatures. In this method, the data variance contribution in principal component will be preserved, and while getting rid of the secondary principal component contributions.

As mentioned above, there are compressed 15 groups signal data for the different damage severities. The principal component eigenvalues are listed in Table 1, together with their relative and cumulative percentages. It is observed that the first 3 eigenvalues account for almost $100 \%$ of cumulative percentages. Therefore, the reconstruction of the impedance data using 3 principal components (PC) will represent a reduction of 200:3 and the error would be almost zero.

Table 1. Principal component characteristic value and the cumulative percentage of compressed data

\begin{tabular}{clll}
\hline Order number & Characteristic value of PCs & Accumulated value & Cumulative percentage \\
\hline 1 & 987.27000475 & 987.27000475 & 0.98708160 \\
2 & 12.83012182 & 1000.10012657 & 0.99990928 \\
3 & 0.02883750 & 1000.12896407 & 0.99993811 \\
\hline
\end{tabular}

Table2 Specification of damage type and output data

\begin{tabular}{ccc}
\hline Damage category & Sample data sources & Output \\
\hline Non-damaged & Pristine & $(1,0,0)$ \\
Slightly damaged & Loosening No.1 or No.1 and 2 bolts & $(0,1,0)$ \\
Serious damaged & Loosening No.1, 2 and 3 or No.1, 2, 3 and 4 bolts & $(0,0,1)$ \\
\hline
\end{tabular}


BP neural network has very strong adaptive capacity, and a very good ability to nonlinear modeling. BP-ANN can overcome the difficulties of inconformity by solving the inverse vibration problem [6]. Therefore, the combination of PCA and BP-ANN can show their respective advantages, and will be well applied to the structural damage detection. According to the above analysis, the use of 3 principal components is considered to be adequate. 9 rows of the compressed impedance data are used as input samples for training the network and the other six rows of data are remained for damage detection. The specification of damage type and output data are shown in Table 2.

After successful training and verification of the network, the other 6 rows of compressed impedance data are fed sequentially into the appropriate network. The output from the network is listed in the following. Simulations are seen to be successful for damage detection, the output yielding a clear distinction.

Test $=$

Columns 1 through 3

Columns 4 through 6

$\begin{array}{lll}0.118451574604246 & 0.036853580089716 & 0.047545182003615 \\ 0.999821026971204 & 0.960046319944553 & 0.938449212948484 \\ 0.000019828743200 & 0.024763174227850 & 0.008427186743997\end{array}$

$\begin{array}{lll}0.329388329377938 & 0.184102376238888 & 0.233701455794906 \\ 0.000000000000000 & 0.002300187289283 & 0.002175173550074 \\ 0.992361153006534 & 0.985224101651338 & 0.978378440640461\end{array}$

\section{Conclusions}

In this paper, an investigation based on experimental data is presented using ANN and EMI technique to detect damages in the steel frame. First, the compression sensing theory is used to reduce the dimension of the conductivity data. The dimension of the compressed data is only $40 \%$ of the original ones and also keeps the initial damage information. Then, a principal component analysis (PCA)-based data reduction technique is further applied to the compressed EMI data. The obtained principal components (PCs), are then used as ANN input variables instead of the pristine EMI data. The results indicate that the combination of EMI data reduction via the compression sensing theory, the principal component analysis and the use of artificial neural networks provide a suitable method for damage detection.

\section{Acknowledgements}

This work was financially supported by the Zhejiang Provincial Natural Science Foundation of China (No. LY12E08005) and the Impact and Safety of Coastal Engineering Initiative, a COE Program of Zhejiang Provincial Government at Ningbo University (No. zj1216).

\section{References}

[1] Park G , Inman D J. Structural health monitoring using piezoelectric impedance measurements. Philosophical Transactions of the Royal Society, 2007, 365: 373-392.

[2] Cai Jin-biao, Chen Yong, Yan Wei. Three-dimensional finite element analysis based electromechanical impedance model and its application. Journal of Zhejiang University: Engineering Science, 2010, 44(12): 2342-2347.

[3] Candes E J, Wakin M B, An introduction to compressive sampling, IEEE Signal Processing Magazine, 2008, 25(2): 21-30.

[4] Candes E J, Tao T. Decoding by linear programming, IEEE Transactions on Information Theory, 2007, 53(12): 4655-4666.

[5] Jin Jian, Gu Yuan-tao, Mei Shun-liang. An Introduction to Compressive Sampling and Its Applications. Journal of Electronics \& Information Technology, 2010, 32(2): 470-475. 
[6] Ma Xiang-sen, Shi Zhi-yu. Structural damage localization based on GA-BP neural network. Journal of Vibration Engineering, 2004， 17(4): 453-456. 